\title{
Pupil Plane Interferometry: Some Conclusions from SCASIS
}

\author{
C.M. de Vos ${ }^{*}$, , J.D. Bregman ${ }^{*}$ and U.J. Schwarz ${ }^{\circ}$ \\ - NFRA, P.O. Box 2, 7990 AA Dwingeloo \\ - Kapteyn Laboratory, P.O. Box 800, 9700 AV, Groningen
}

\begin{abstract}
In this contribution we summarize some conclusions from the SCASIS project. The project, which concerns a multiple shearing pupil plane interferometer, was started in 1986 and was concluded in 1992 with the PhD thesis of de Vos.
\end{abstract}

The SCASIS project was intended as a feasibility study for the application of a redundant multiple shearing pupil plane interferometer. We will now summarize the results of that study.

In the first place it turned out to be feasible to construct a redundant multiple shearing interferometer which allows us to use most of the light collected by a large telescope (see Figure 1). The final prototype includes options for atmospheric zooming and monitoring of the seeing. However, the special segmented lenses used, and the amount of supporting optics needed for the pupil plane configuration, make the optical layout complicated. Setup and alignment are difficult, and need to be checked repeatedly. The instrument cannot be left on the telescope for general use. Initially we used an eight aperture system. The reduction of several objects showed that it is much safer to work with a five aperture system, which is still over-redundant, but gives more pronounced visibility peaks. This allows for more accurate calibration and in particular makes the correction for the photon bias less critical.

Secondly, it turned out that the proposed video detector system, using a CCD camera and two Intensified TV cameras was feasible for the observations. The detective quantum efficiency of the intensified cameras allows observations of objects down to $6^{\text {th }}$ magnitude. Initially, the darkcurrent turned out to be too high to observe the faintest sources. An appropriate reduction of the darkcurrent could be achieved with a simple cooling system. The storage on video tape allows efficient storage of large quantities of data, with sufficient accuracy in terms of geometric distortions, contrast degradations and stability of eventshapes. Large numbers of video frames can be digitised and prepared for processing using the multi-pass retrieval system. The fixed integration time turned out to be not critical in terms of limiting magnitude, but made it impossible to get high signal to noise in single frames. Detector systems put severe limitations on optical interferometers, mainly in terms of quantum efficiency and local dynamic range. Comparison of the video system with the digital IPD system shows that the video system is in many aspects better suited for use in an interferometer like SCASIS (with isolated visibility peaks). The countrate is much higher and the MTF is slightly better. The intensified cameras do not suffer from 
the unrecoverable non-linear effects caused by eventcentring hardware. The additional advantage of on-line moving pictures of pupil and image plane images should not be underestimated. During the alignment of a complex instrument, such direct views are vital. Also, since atmospheric conditions can vary on short timescales, a direct visual impression of the observed material is very helpful for scheduling of observations and selection of recorded material. We were able to develop additional tools to quantify such impressions, in terms of seeing quality and sharpened images.

In the third place, we were able to develop and code the proper algorithms for the reduction of the measured SCASIS fringes. In particular, we succeeded in making a proper correction for the photon bias in fringe visibilities and closure phases and for the line triples that we calculate in the reduction of spectral observations. We found various ways to verify the corrections. Instrumental effects could be successfully removed with a point source calibration. Unfortunately, it turned out that delay effects, introduced by the segmented optics, limit our effective integration area more severely than atmospheric turbulence. So we use the flexibility allowed by the pupil plane concept to correct for the instrumental effects. Point source calibration does not cancel the decorrelation over the integration time of the cameras, if the seeing changed during the observations. This introduces a slight dependency on atmospheric conditions that is not removed by the redundancy. It can be removed in the hybrid mapping procedure used for the image reconstruction. The effect results mainly in a lower dynamic range in the final images.

With these boundary conditions of optical instrumentation, detector and data acquisition system and reduction software set, we could study the feasibility of the instrument for actual observations. The ability of a pupil plane configuration to match the coherence area at the reduction stage turned out to be not critical in terms of limiting magnitude. We could still use the matching to get the same known amount of decorrelation in different observations. The other main advantage of the pupil plane system is the option to track seeing cells as they move over the pupil, thus extending the effective integration time. This requires zooming on a dominant layer of turbulence. We found strong indications that multiple dominant layers are present most of the time. We verified this in a specific experiment where we found indeed two well separated layers of turbulence, with different properties. Although the lifetime of seeing cells was shown to be larger than the coherence time (a necessary condition for seeing cell tracking) we concluded that seeing cell tracking cannot be generally used. Making images of stellar objects, we suffered from additional decorrelations. The main sources were found to be delay errors in the optics, atmospheric turbulence within the fixed integration time and (in some cases) smearing of integrated visibility peak due to wavefront tilts over the apertures. We were able to construct images with more than 1:40 dynamic range for a 3.6 magnitude star, with a five aperture configuration. Imaging of a fainter system (components closer than 0.2 arcsec, total magnitude 4.5) in an eight aperture configuration gave much worse signal to noise, but produced results with a dynamic range of 1:10 (see Figure 2). This lower value is explained by the loss of contrast due to the larger number of strong components in the source and due to the fact that we used more apertures. It turned out that a suffiently redundant set of closure phases was essential to reconstruct this triple source (with three roughly equal $5^{\text {th }}$ magnitude components) unambiguously.

Summarizing we can say that the instrument has been shown to work indeed. Some of the theoretical advantages of the pupil plane approach turn out to be of less practical value, due to instrumental effects and the nature of atmospheric turbulence. The pupil plane 
system is in principle well suited for high resolution observations. The complicated optics make it difficult to operate. Although none of the many optical elements (apart from the segmented lenses) is critical, it is very difficult to verify the influence of each individual element experimentally.

More objects have been observed already. Preliminary examination shows that the observational material is of sufficient quality to allow for proper reduction and image reconstruction. We are currently processing observations of $\mathrm{P}$ Cygni, for which we earlier found indications of asymmetry on intermediate resolutions.

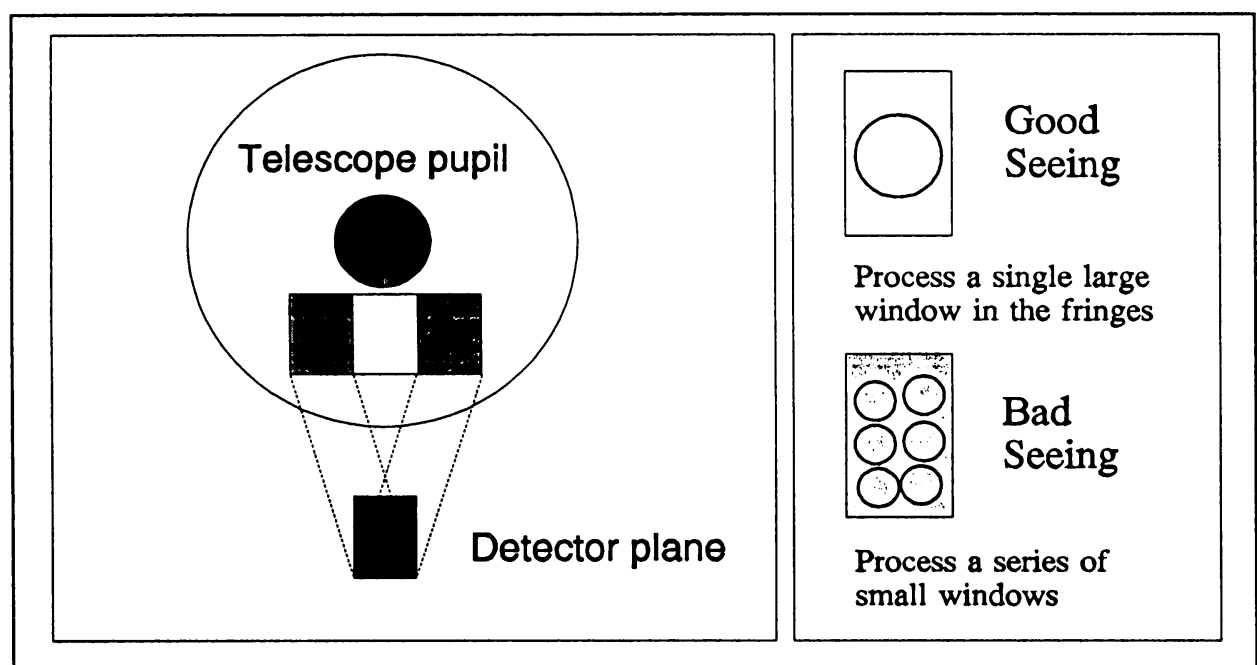

Figure 1: Schematic representation of a multiple shearing pupil plane interferometer, allowing to use most of the telescope collecting area irrespective of seeing conditions.

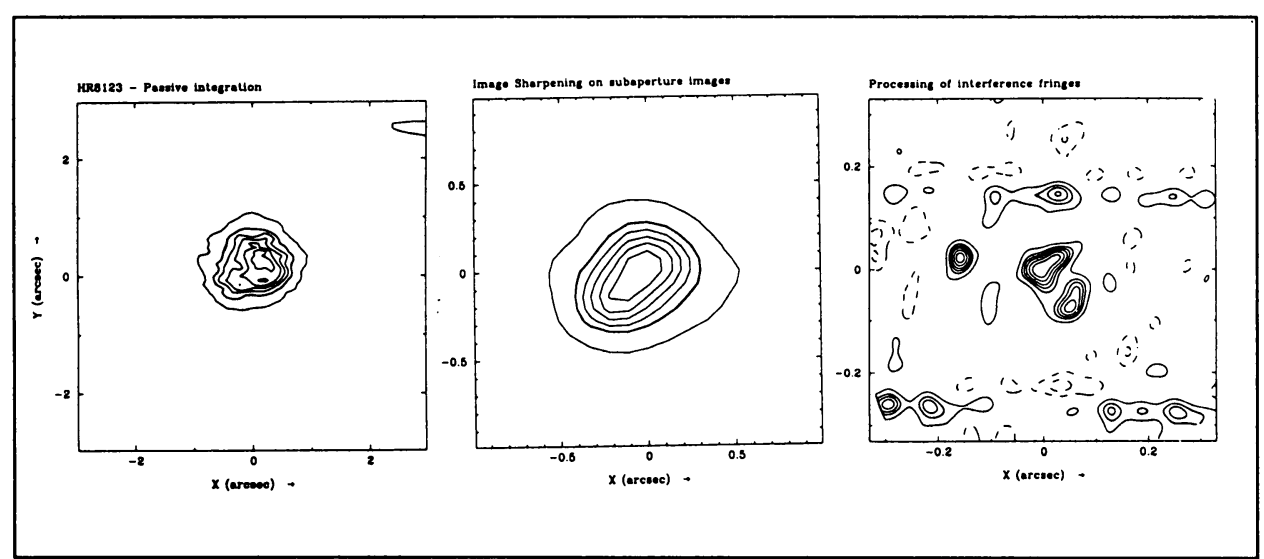

Figure 2: Imaging results for HR8123 based on passive integration of I'TV images (a), Image Sharpening on subaperture images (b) and processing of interference fringes (c). 